\title{
COLD ZONE EXPLORATION USING POSITION OF MAXIMUM NUSSELT NUMBER FOR INCLINED AIR JET COOLING
}

\begin{abstract}
Inclined jet air cooling can be effectively used for cooling of electronics or other such applications. The non-confined air jet is impinged and experimentally investigated on the hot target surface to be cooled, which is placed horizontally. Analysis and evaluations are made by introduction of a jet on the leading edge and investigated for downhill side cooling to identify cold spots. The jet Reynolds number in the range of $2000 \leqslant \operatorname{Re} \leqslant 20000$ is examined with a circular jet for inclination $(\theta)$ of $15<\theta<75$ degree. Also, the consequence of a jet to target distance $(H)$ is explored in the range $0.5 \leqslant H / D \leqslant 6.8$. For 45 degree jet impingement, the maximum Nusselt number is widely spread. Location of maximum Nusselt number is studied, which indicates cold spots identification. At a higher angle ratio, the angle is the dominating parameter compared to the Reynolds Number. Whereas at a lower angle ratio, the inclined jet with a higher Reynolds number is giving the cooling point away from leading edge. It is observed that for a particular angle of incident location of maximum Nusselt Number, measured from leading edge of target, is ahead than that of stagnation point in stated conditions.
\end{abstract}

\section{Introduction}

The challenge is to remove heat from hot objects that may not be easily cooled due to the limitations of cooling techniques. The heat transfer convection limits are specified for various applications [1]. The methods, including passive heat sinks and active heat sinks, are useful as they will typically not have leakage and pipeline issues as compared with liquid cooling. Also they do not need extra energy, called passive techniques [2]. To improve effectiveness, a foam heat sink [3] is also preferred during fluid flow conditions. A conventional vapour compression cycle of refrigerator cooling has high effective cooling, but it is expensive and requires

\footnotetext{
${ }^{1}$ Dr. D. Y. Patil College of Engineering and Innovation, Savitribai Phule Pune University, Pune, India.Email: sbingole1@ rediffmail.com

${ }^{2}$ Savitribai Phule Pune University, Pune, India.Email: vit_sundaram@yahoo.com
} 
more space for the system. Forced air cooling methods have their own applications and are normally used because of their reduced cost, consistency and technical improvements. Air cooling techniques like piezo fans (solid state devices), synthetic jets (periodic micro jets), and nano-lightning (micro scale ion driven, using a very high electric field) are found during various cooling applications. Air cooling systems are deployed in combination with a heat sink. Air touches a heat sink at a significantly high temperature, and it will reduce its capacity to transfer heat. Jet cooling might be the option for the same [4]. Hence, hybrid thermal management solutions are becoming more popular in cases of thermal management problems [2]. The examples can be listed: air - water, sink - air jet, sink - fan, sink - liquid jet, sink - thermoelectric, sink - spreaders, etc. [5]. Also, cross flow systems of similar fluid gives different flow conditions and heat transfer characteristics. Tabulators or inserts are also used in systems with twisted tapes, coil inserts, swirls generators, etc. Liquid cooling, especially with water, may be the most common solution. But even it has good thermal conductivity and a higher heat of evaporation, its dielectric constant is the parameter that prevents it from day to day use. In micro channel cooling, higher heat densities are to be removed [6]. Liquid metal cooling is also used which remains liquid and also has a low coefficient of thermal expansion and can be used for cooling systems up to $200 \mathrm{~W}$ per square $\mathrm{cm}$ [1]. Use of a thermo-siphon and thermoelectric system like a heat pipe is costly. The boiling and liquid forced convection has the highest heat transfer rates, and air cooling is also widely used in many applications due to its availability of fluid, simple systems, and cost effectiveness.

Materials (for making electronics components) and heat distributers (like TIMs and thermal paste) heat spreaders, use of nano-technology [7] are frequently identified. This can be considered as a separate area of research, specially related to material science in electronics cooling. The challenges in thermal management are to design lower volume systems with high heat dissipation at low cost, which may work with a lesser chance of failure. Also, there is a challenge to develop low cost high-conductive adhesive materials, spreaders, use of techniques like heat pipe, vapour chambers, thermoelectric cooling, in combination as hybrid system. Also, the application of direct liquid cooling, spot cooling to cool hot spots in the system, developing high performance air cooling/air movers are the challenges. The component generating heat is generally taken care of by spot cooling systems. Normally, heat can be removed by convective mode, but the issue of hot spot cooling should be tackled properly for any electronics or such systems. Jet cooling can be one of the best solutions for this.

\section{Jet impingement review}

Jet cooling can be well-defined as the impingement of high velocity fluid on the target surface to be cooled through an opening/pipe/nozzle. Jet impingement fluids mostly used are air or high heat transfer coefficient liquids. The jets can be 
classified as per different criterion viz. based on fluid flow [8], based on inclination of jet and based on orientation of jet. Air jets have the capability to dissipate high heat flux, and they can also cool hot spots. The jet cooling can have advantages, as they can cool up to 90 to $180 \mathrm{~W} / \mathrm{m}^{2}$. The fluids, as air and water, are easily available at low cost.

They are useful for hot spots to be cooled on the target. Even and uniform cooling can be achieved, and these can be installed on an available entire system directly. The disadvantages are that it needs pump/micro pump/blower/compressor with extra power. Also blocking/chocking can be a problem for micro jets with manufacturing difficulty. The noise level of fluid impingement and difficulty in installing for portable electronics devices can be major issues.

It is possible to use various physical structures for jet cooling. Varieties of combinations are found in the experimentations by changing design of flow channel, swirl generation, exit vent provisions, vibrations, etc. [9-11]. Different fluids can be used for carrying away heat from a hot source. The type of fluid depends on different features of the system to be designed for cooling, such as the specific application, type of component to be cooled, size, system temperature range, cooling needs, heat generation parameters, space available for cooling system; additional energy needed and permitted for cooling system, etc. [12, 13].

Comparison of heat transfer to single, axisymmetric, submerged and confined air and water jets is found and can be used directly [14]. Instead of a single fluid, the use of mist fluid on heated metal surfaces, such as water-air mist, can be done to get other characteristics [15]. If fluid is also one of the important selection constraints for the jet, for economic considerations, the method of generating jets is also considered to be significant. Synthetic jets are used classically for saving space, but have some restrictions. Normally, jets are established by pressurizing the fluid. Air jet produced by compressed air, water jet produced by water pump, but synthetic jets uses electromagnetic principle (by which micro-jets are produced) $[16,17]$. The structural exit cross-section of the jet gives varied flow patterns of the jet, which has an effect on cooling ability. It is also true that its practical use is dependent on ease in manufacturing of the jet [18-20]. It is observed that slot jet and circular jets are used regularly due to their simplicity in manufacturing and applications. The frequency of the jet designates the magnitude of the pulsation of the jet. Pulsating flow increases mixing, which increases turbulence and then heat transfer. Such jets are efficient with saving energy, and for their special flow patterns generated which create turbulence and enhance heat transfer [21, 22].

It is also observed that different types of jets have their own advantages and fluid flow patterns, but flow currents, eddies and ultimately heat transfer also depend on the surface on which the jet is to be impinged. The target surface also directs and controls enhancement of heat transfer [23, 24]. To make cooling systems squeezed, the cooling jets can be inclined [25]. The cooling effectiveness is a function of the Reynolds number, characteristic length of plate to be cooled, 
point of jet impingement, and the oblique angle of the inclination of the jet. Cooling using sprays [26] and micro-jets, using nozzles for droplet sprays, orifice plates to create arrays of micro jets, can also be used. By changing various parameters, efforts are made to know the physics of the flow, and variations in heat transfer using jets can be listed as follows: velocity of jet, diameter of jet, angle of impact, number of jets, and jet to target spacing, jet to jet spacing in the case of a greater number of jets, turbulence levels, jet cross section, jet length, confinement of jet, target surface, and fluid properties.

In an inclined jet also, the cross section area of the jet is an important parameter, and variations in heat transfer characteristics are seen [27]. The maximum heat transfer coefficient decreases with the increasing of the jet angle and its position changes towards the upstream direction. Two jets with different geometry and at different Reynolds Numbers may lead to acquire more turbulent intensity. This shifts stagnation point back and forth and lead to increase effective cooling area [28]. Cross flow impingement [29], spray cooling [30] downward facing inclined wall [31] are also investigated. The hot object which is to be cooled is placed in a moving position rather than stationary [32], and it is impinged with the inclined jet $[33,34]$.

The objective of the current study is to examine and recognize the consequence of offset jet impingement cooling by using an inclined air jet at Reynolds numbers of 2000 to 20000 , and also to incorporate and propose correlations for the position of maximum Nusselt Number using inclined air jet for offset cooling and to investigate the effect of "target to jet distance" with different angles for designing cooling systems with cold spot positioning.

The scope is limited to the range of the experiment for the diameter of jet $(D)$, angle of impingement $(\theta)$, target to jet distance $(H)$ and velocity of fluid impingement $(V)$.

\section{Terminology of inclined jet}

A two dimensional jet ( $X$ and $Y$ directions) can be considered to know the terminology used. The jets are impinged through the pipe of diameter $D$ on a hot target surface to be cooled. The ambient air is used as fluid in this case. If $V_{0}$ is the velocity of fluid flow at the exit of the jet (whereas, $V_{m}$ is maximum velocity of jet during travel), it mixes aggressively with the nearby fluid which generates turbulence, and the size of the jet increases by making it thicker. The terms like potential core, maximum velocity achieved at the centre are well known and shown in Fig. 1. Jet spreading generates a larger lateral velocity gradient by which the velocity increases. Near the jet opening, a mixing region develops a potential core, and turbulent exchange of heat and mass takes place. In the potential core, properties of fluid are relatively constant. At the end, the core is dissolved because of the mixing action. The length of potential core during jet impingement is 5 times the width of the slot jet $[35,36]$. 


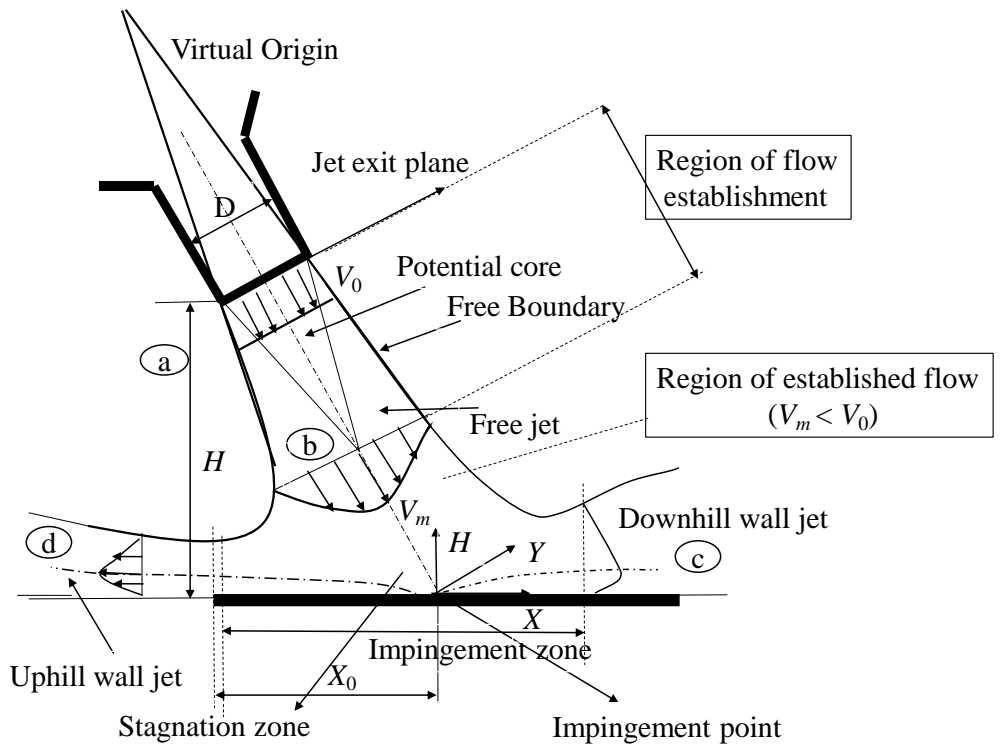

Fig. 1. Terminology of jet impingement

The next zone is of fully developed flow region and is developed due to a large penetration of turbulence to the axis of the jet, by which the potential core dissolves, called a "free jet". The mean velocity on the axis starts to decline with development. The added dragging of fluid in the jet increases its mass flow. During the inclined impingement of the jet, the point where the jet axis intersects with the target surface is called the impingement point/geometrical stagnation point. The distance along the $X$ axis at which the geometrical point lies is defined as $X_{0}$. In the geometry of Fig. 1, $H$ is the height from the lowest opening edge of the jet to the target plate. It is specifically defined, as it is easy to measure it physically [36]. The impingement zone is the zone shown between two vertical lines, which is asymmetric. After impingement, it will be turned in a positive and negative direction where the flow is accelerated again. The conservation of momentum principle will increase the velocity of the wall jet after the flow diverges by turning and growth of the boundary layer.

The diverted flow is decelerated again drastically, which is termed as a downhill wall jet. The wall jet has a minimum thickness of 0.75 to $3 D$ for the perpendicular jet, and then it gets wider as it progresses away from the jet.

In the case of an inclined jet, the influencing parameters can be expressed as follows: In case of an inclined jet, the influencing parameters can be expressed as follows: hydraulic diameter of jet $(D)$, target to jet height $(H)$, convective heat transfer coefficient $(h)$, thermal conductivity of fluid $(k)$, velocity of fluid coming out of jet $(V)$, kinematic viscosity $(\mu)$, density of fluid $(\rho)$, specific heat $\left(C_{p}\right)$, temperature difference $(\Delta T)$, geometrical stagnation distance measured from leading 
edge $\left(X_{0}\right)$. Hence, it can be written as [37]:

$$
f\left(D, H, h, k, V, \mu, \rho, C_{p}, \Delta T, X_{0}\right)=0 .
$$

If the parameters are studied, they can be reduced to non-dimensional terms with Nusselt number (Nu), Reynolds number ( $\mathrm{Re})$ and Prandtl number (Pr) as:

$$
\mathrm{Nu}=f\left(\frac{H}{D}, \operatorname{Re}, \operatorname{Pr}, \frac{X_{0}}{D}\right) .
$$

\section{Methodology and Experimental Setup}

In the view of analysis of the parameters decided for investigation, experimentation is proposed. The experiment consists of a jet arrangement with air as the fluid. The target plate is to be heated by supplying electrical energy that is recorded by measuring voltage and current. This energy is supplied to the target plate for heating, which is to be carried out by fluid flow of the impinged jet [36]. The heat energy can be passed out through heat transfer from the bottom and top of the plate majority by convection, and also by conduction and radiation. The heat transfer through conduction by thin target is very small and hence can be neglected. The radiation heat loss by the thin foiled target from the bottom and top side is calculated by using conventional methods. Correction factor is applied during analysis.

The energy coming out of plate is written as:

$$
E_{\mathrm{o}}=Q_{\text {conv. } \mathrm{j}}+Q_{\text {conv.b }}+Q_{\text {rad.t.b }}+Q_{\text {cond }}
$$

where, $E_{\mathrm{o}}$ - total energy coming out, $Q_{\text {conv.j }}$ - heat transfer due to convection by air jet, $Q_{\text {conv.b }}$ - heat transfer by convection from bottom side of plate, $Q_{\text {rad.t.b }}$ - heat radiated by top and bottom surfaces, and $Q_{\text {cond }}$ - heat conducted through plate. The Reynolds number is calculated on the basis of the diameter of the jet that indicates

$$
\operatorname{Re}_{D}=\frac{\rho V D}{\mu}
$$

By using these parameters, the local Nusselt number and average Nusselt number are calculated at every location of the plate. The experimental setup is as shown in Fig. 2.

The arrangement for production of the air jet and its impingement on the target are made. The target surface is positioned as horizontal. The test target is a hot, plane and smooth plate, made up of a thin foil of stainless steel with a thickness of $0.050 \mathrm{~mm}$ and heated by the Joule effect and one provided arrangement for tightening the foil using housing of target plate, as in Fig. 3 [38].

The housing is made with the intention of placing the target plate in the required position. Two copper bus bars are used with the heater plate foil. The 


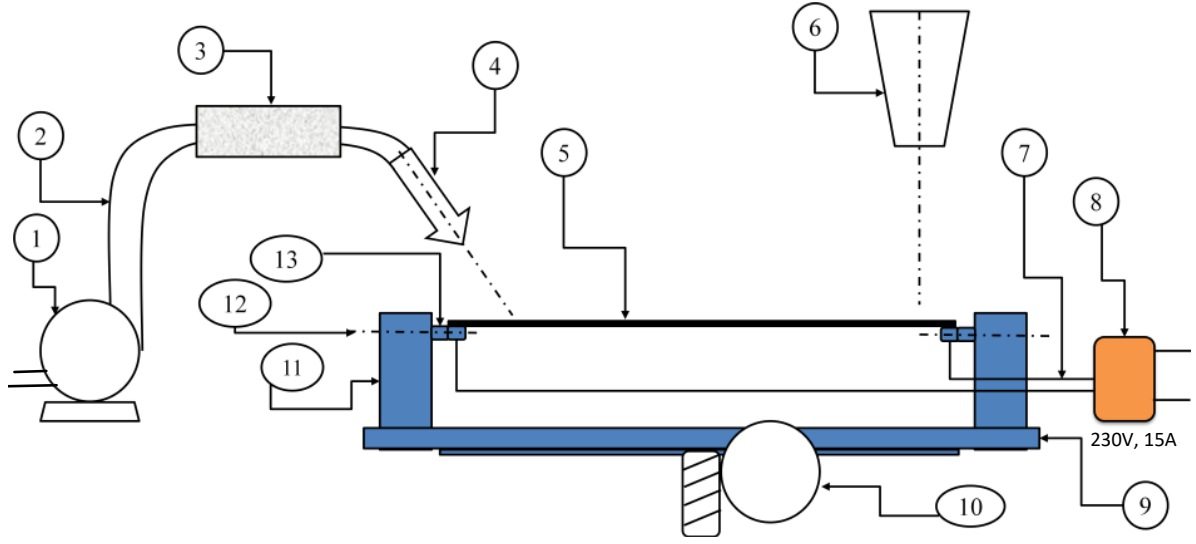

Fig. 2. Block diagram of experimental setup: 1 - blower for air supply, 2 - hose pipe, 3 - air cooler, 4 - inclined replaceable jet, 5 - target plate, 6 - infrared camera, 7 - electrical cables,

8 - transformer, 9 - base housing, 10 - hand wheel for changing height, 11 - vertical support, 12 - tightening bolts, 13 - bus bars

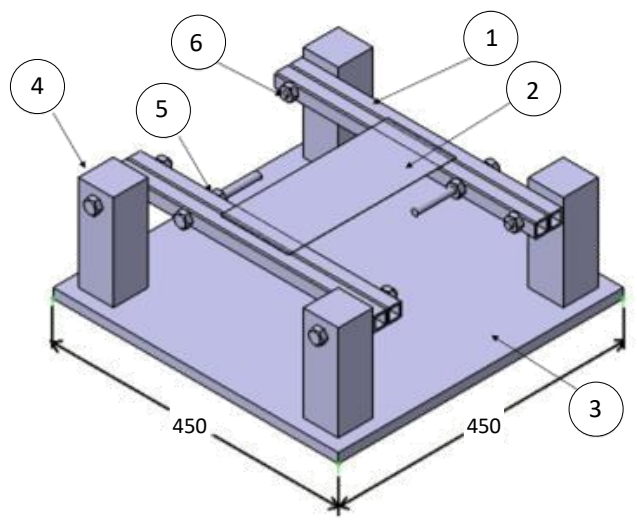

Fig. 3. Housing of a target plate: 1 - bus bars, 2 - target surface, 3 - base, 4 - support, 5 - foil fixing bolts, 6 - expansion correction bolts

supply of $230 \mathrm{~V}, 5 \mathrm{~A}$ is given to a transformer and converted to the required ratings of 3 Volt and 50 Ampere AC. This heated target plate is cooled by impinging air through a circular cross-section jet. The width of the target plate (shorter side) is considered as the reference $Y$ axis for locating the jet position. The jet is placed at the centre position along the width of target surface. The longer side of the target plate (length) is considered as the $X$ reference axis [36]. This is also satisfying requirements of air at Reynolds number varying from 2000 to 20000. To understand the effect of a change in height $H$, it is varied from $10 \mathrm{~mm}$ to $55 \mathrm{~mm}$. The axis of the jet can make an angle of 0 to 90 degree with the horizontal target. An infrared thermometer is used to take temperature readings of the target 
plate. The velocity of the air jet is measured in $\mathrm{m} / \mathrm{s}$ by using an anemometer. All readings are taken at steady state conditions. The jet diameter is considered as the characteristics dimension, which is comparatively simpler and widely accepted way of study.

\section{Results and discussions}

Analysis of the inclined jet is performed by considering various criteria of the investigation. The parameters selected for analysis are: Nusselt number distribution along $X$ direction and location of maximum Nusselt number.

\subsection{Nusselt number distribution - effect of variation in Re and H/D}

As stated and explained in earlier sections, readings are taken at various Reynolds numbers with the aim of calculating the Nusselt number along $X$ direction of the target plate. The local Nusselt number is a function of temperature, indicating temperature as an important parameter. Average temperatures along the dividing lines on the target plate are calculated and plotted along $X$ axis to understand variation in Nusselt number. The local $\mathrm{Nu}$ distribution along $Y$ axis is following symmetry about the centreline of the plate. The symmetry is seen to be unaffected to change in jet inclination [39]. Hence, the analysis is performed by the same claim. It is a clear fact that the Nusselt number increases as per Reynolds number in agreement with [25]. The analytical solution can be worked out for laminar jets. However, given the fact that it gives a lower range of heat transfer, a turbulent range of Reynolds number is selected for this study. Some sample cases are shown in this section in Fig. 4. Similar trend lines are observed for a typical combination of parameters. But there is not much more change in pattern of heat transfer distribution with Reynolds number along the $X$ axis.

The effect of variations of $H / D$ in relation with the average $\mathrm{Nu}$ along $X$ axis of target plate is shown (sample case - for a jet diameter of $8 \mathrm{~mm}$ and jet inclination of 45 degree) in Fig. 4. As the inclination angle decreases, the coolest points with heat transfer shift to the downhill side of the target plate. At a higher $H / D$, the jet delays itself to reach the target because of the higher value of $H$. Due to time lag, the size of the vortex increases, but at the same time its energy decreases. As a result, the vortices produced in the jet collapse as and when they reach the potential core, before the jet reaches the target plate. This in turn shifts the $\mathrm{Nu}_{\text {avg }}$ profile, showing a decreasing trend with an increase in $H / D$. The highest $\mathrm{Nu}$ is observed for $(X / D)$ of $8,11,12$ and 12 for $(H / D)$ of $1.25,3.13,5$ and 6.88 respectively. Whereas, the jet jump is seen in an uphill side typically for $(H / D)$ of higher than 5 giving hot spot at $(X / D)$ of 7 in the present case. All Nu lines will merge as $(X / D)$ increases, and it depends on Re. 


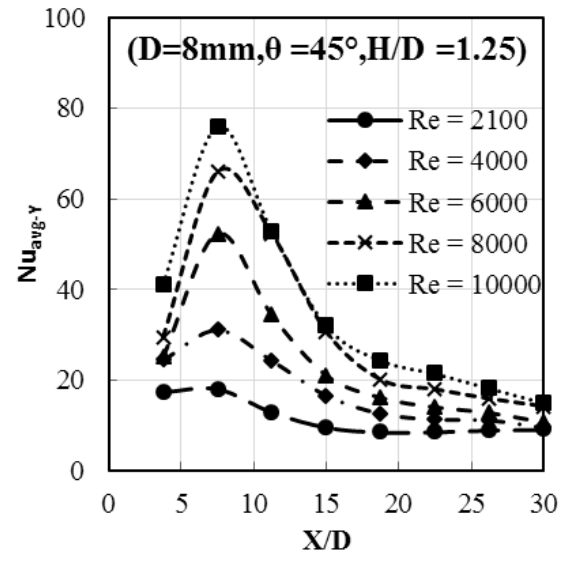

(a) $H / D=1.25$

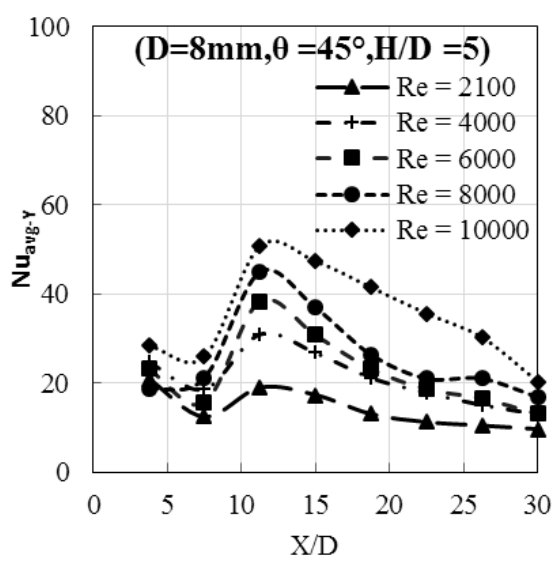

(c) $H / D=5$

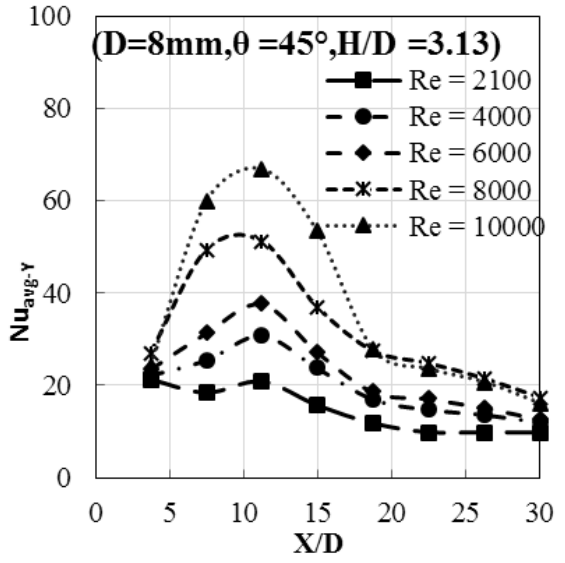

(b) $H / D=3.13$

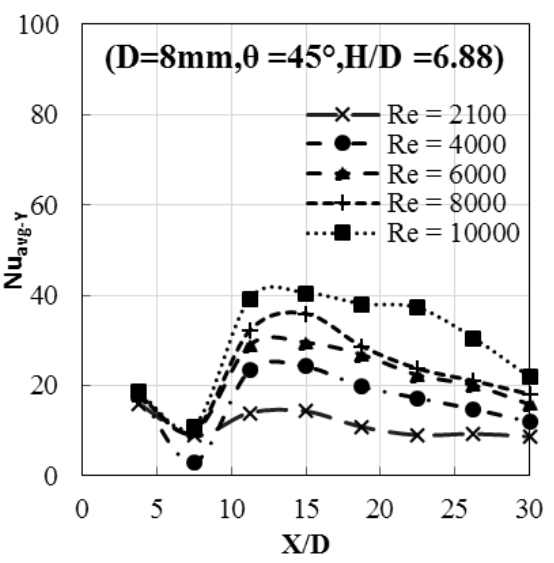

(d) $H / D=6.88$

Fig. 4. Average Nu w.r.t. Reynolds number for various $H / D\left(D=8 \mathrm{~mm}, \theta=45^{\circ}\right)$

\subsection{Nusselt number distribution - effect of variation of inclination}

To study the effect of variation of the inclination of a jet on a target surface, plots are drawn by keeping all other parameters constant, like the jet diameter of $8 \mathrm{~mm}$ and $(H / D)$ as 5, as shown in Fig. 5. The highest heat transfer occurs at $X / D$ of 5, 7, 12 and 22 for jet inclinations of 75, 60, 45 and 30 degree, respectively. As the Reynolds number decreases, the Nusselt number also decreases along the $X$ direction. A jet inclination angle decreases, at around 60 degrees, the upstream and downstream flow Nusselt number spreading becomes approximately symmetrical [35]. However, in the present case it is not showing symmetrical patterns.

On the contrary, the downward side is giving variations according to the Reynolds number, and the uphill side profiles remain nearly constant giving a 


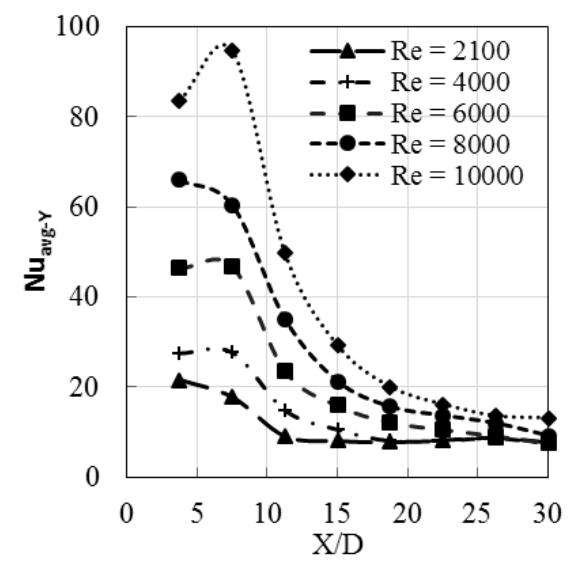

(a) $75^{\circ}$

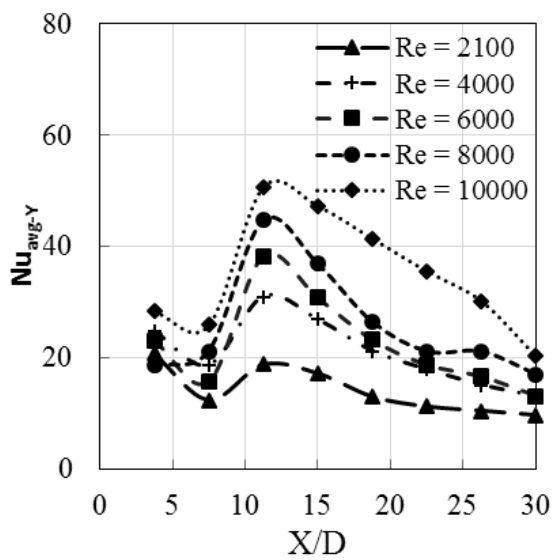

(c) $45^{\circ}$

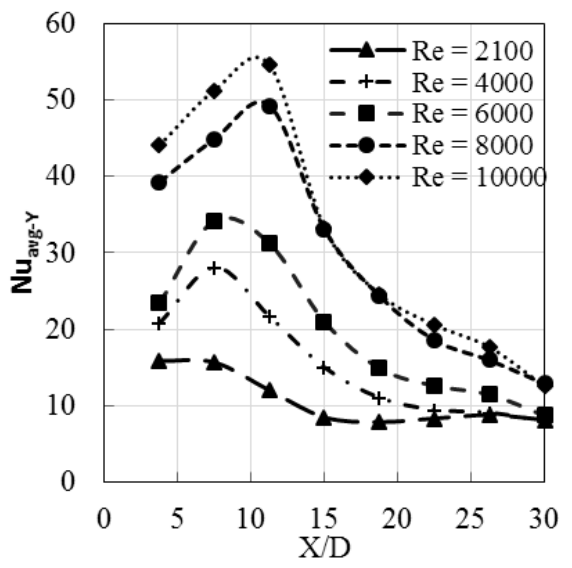

(b) $60^{\circ}$

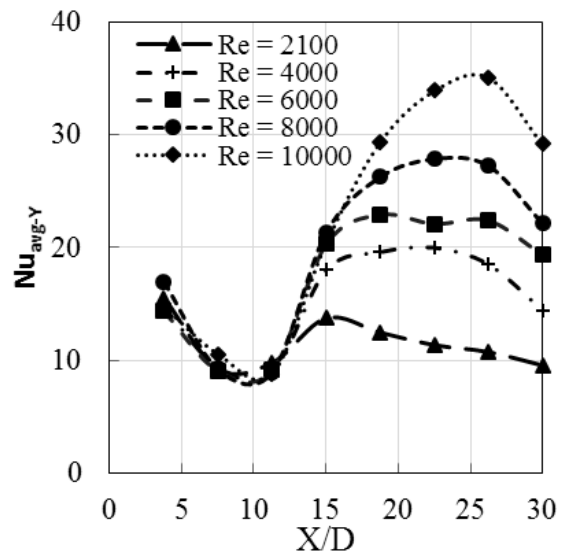

(d) $30^{\circ}$

Fig. 5. $X$ direction Nusselt number for $D=8, H / D=5$ with variations jet inclinations

lower Nusselt number. In these plots, also the hydraulic jump is seen in the uphill side, as stated in an earlier section. This happens due to the pressure variation in the jet flow and will effect on turn in direction of the jet flow, by variation in shear layer. The flow is decelerated and generates normal stress and strains which supports turbulence in jets [40]. The turbulent flow in a jet has large vortices, which may divert themselves into the boundary layer of the flow and exchange fluid of dissimilar thermodynamic properties. The capability of these basic vortices to brush away the boundary layer as the jet flows against the wall increases the local Nusselt number. The lines of $\mathrm{Nu}$ are collapsed and form one line in the uphill side of the jet below $(X / D)$ of 12 for a 30 degree jet inclination in the present case giving uniform cooling for all Reynolds number. 


\subsection{Position of maximum Nusselt number - inclination wise}

The average Nusselt number and maximum Nusselt number are analyzed. At the same time, it is important to know the location of this maximum Nusselt number on the target plate. It will be of interest to know the position of this number along the $X$ axis in the direction of flow. The symmetry along the centreline on the target permits this. Hence the next parameter to be examined is the position of maximum Nusselt number. It is defined along the $X$ axis which gives a maximum $\mathrm{Nu}$ and is indicated as $\left(X_{\text {Nu.max }}\right)$. The analysis is performed by understanding the aim of analyzing the entire plate for comprehensive cooling. The position of the coolest point indicates maximum Nusselt number for that configuration of the inclined jet. Fig. 6 shows variation of position of maximum Nusselt number against $\operatorname{Re}(H / D)$.

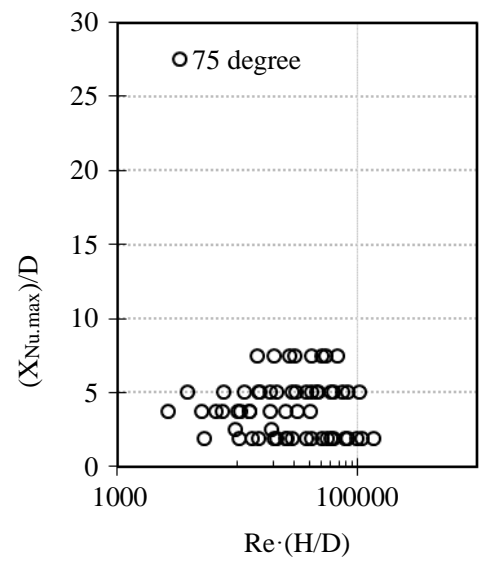

(a) $75^{\circ}$

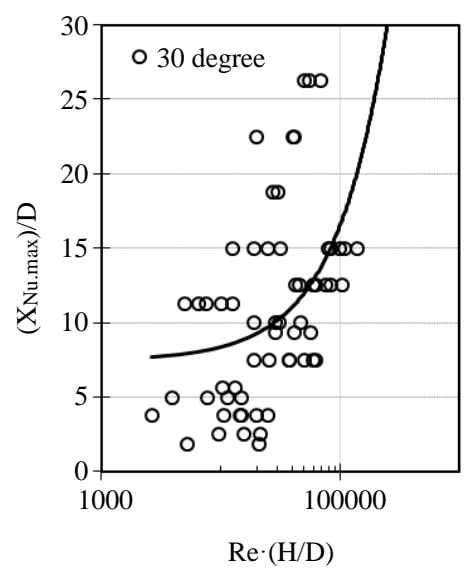

(c) $45^{\circ}$

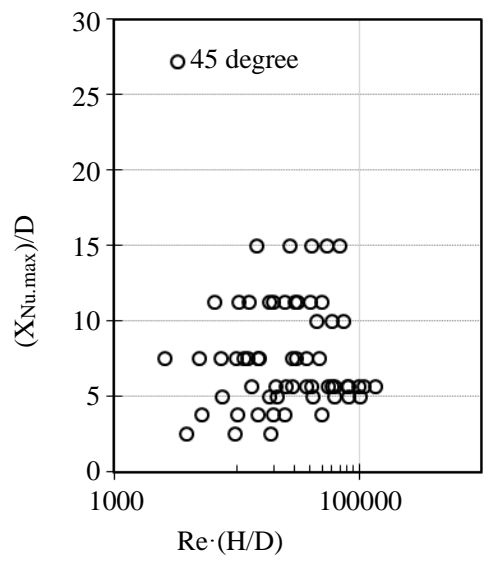

(b) $60^{\circ}$

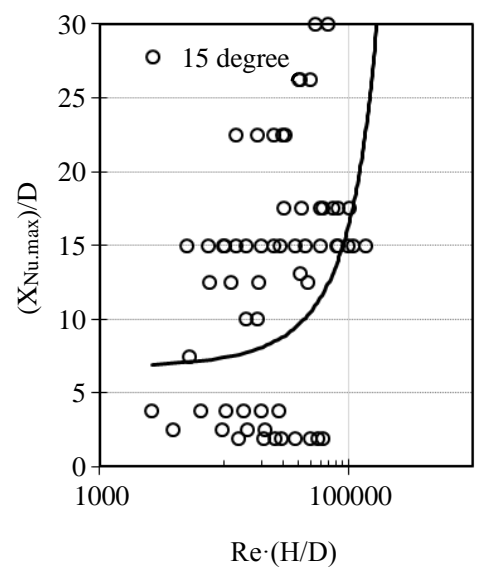

(d) $30^{\circ}$

Fig. 6. $X_{\text {Nu.max }}$ positions for different jet inclinations 
For a 75 degree inclined jet, the Numax location i.e. $X_{\text {Nu.max }}$ is varying from 30 to $60 \mathrm{~mm}$ starting from the leading edge, and it increases up to $250 \mathrm{~mm}$ as Re and/or $H / D$ rises. To normalize data for all diameters, the ratio of the distance of the maximum Nusselt number $\left(X_{\text {Nu.max }}\right)$ and jet diameter $(D)$ is plotted on the $Y$ axis. For the jet inclination of 75 degrees it goes to a maximum 7 , whereas for a 45 degree jet inclination it increases to 15 .

However, for a 30 and 15 degree inclination, it drastically increases to a highest of 25-30. The physics of the flow can tend towards a geometrical intersection point of the inclined jet axis to the target plate $\left(X_{0}\right)$. The angle ratio $(A R)$ is defined as the ratio of actual jet inclination angle $\left(\theta\right.$ or $\left.\theta_{a}\right)$ in degree to perpendicular jet inclination angle $\left(\theta_{p}\right)$ in degree (i.e. 90 degree), mathematically $\left(\theta / \theta_{p}\right.$ or $\left.\theta_{a} / \theta_{p}\right)$. At a higher angle ratio $(A R)$, the angle is the dominating parameter, particularly for the analysis of the location of the maximum cooling position. However, at a lower angle ratio, the effect of the target to jet height, Reynolds number and inclinations are dominating each other; hence it will be difficult to get exact position of maximum Nusselt number. At a lower angle ratio, the inclined jet with a higher Reynolds number is giving the cold point away from the leading edge. The same configuration with a lesser Reynolds number gives a smaller $X_{\text {Nu.max }}$, which is nearer to the leading edge. For comparison of jet inclinations of 30 degree and 15 degree, trends of variation are shown, which indicates shift and agrees with [41]. Also, the Nusselt number increases with the Reynolds number, but the maximum Nusselt number position remains unchanged for a specific angle and target to jet height [35].

\subsection{Location of maximum Nusselt number w.r.t. Reynolds number, angle ratio and target to jet height}

The three important parameters which affect the maximum Nusselt number location are Reynolds number $(\operatorname{Re})$, target to jet height $(H)$, inclination of jet $(\theta)$. These three parameters are correlated using regression analysis. Fig. 7 shows variation between the three parameters.

The correlation suggested is:

$$
\frac{X_{\max }}{H}=0.2957 \operatorname{Re}^{0.1885}\left(\frac{\theta}{\theta_{p}}\right)^{-0.919}
$$

Temperatures on the target plate are measured after every fixed location on the surface $(X=15 \mathrm{~mm}, 30 \mathrm{~mm}, 45 \mathrm{~mm} \ldots)$ with the aim of a comprehensive cooling solution to the problem. This will not give an exact location of maximum Nusselt number or higher heat transfer. Also, the exact location is difficult to obtain without micro analysis. However, by looking towards the cooling of the entire plate, predictions are made [41]. 


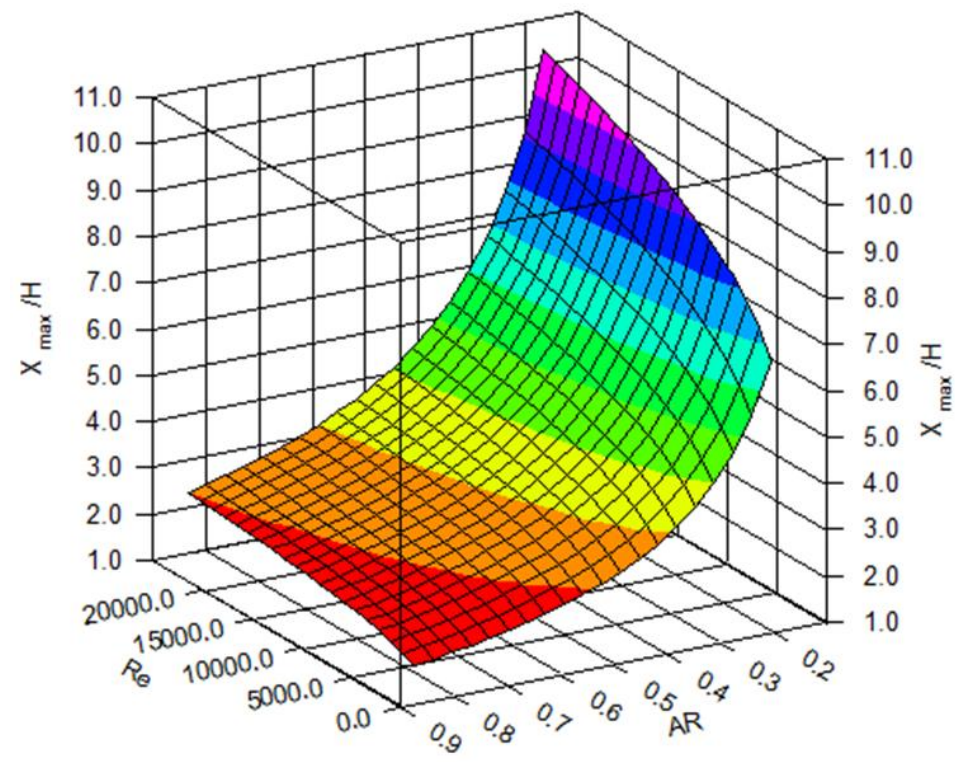

Fig. 7. $X_{\text {Nu.max }}$ - variations between Reynolds Number $(\operatorname{Re})$, target to jet height $(H)$, and Angle Ratio $(A R)$

\subsection{Overall position of maximum Nusselt number}

To understand the position of the maximum Nusselt Number in relation to the variation of the jet inclination, $\theta$ is plotted against $\left(X_{\mathrm{Nu} \text {.max }} / H\right)$. In this $X_{\mathrm{Nu} \text {.max }}$ is the average of all the set of experiments in the range for a particular angle. Almost 100 experimental points are plotted in Fig. 8a). This also clearly proves that, as the angle of inclination increases, the slope to the curve decreases indicating that $X_{\text {Nu.max }}$ shifts closer toward the leading edge. Fig. 8b) shows the variation of the results of $\left(X_{\max } / H\right)$ by comparison using experimental data and data obtained by the equation in this section.

The geometrical stagnation point $\left(X_{0}\right)$ is calculated using simple geometry construction and compared with the location of the maximum Nusselt number. It is observed that the location of cold spots (maximum Nusselt number) for a particular angle of impingement, measured from the leading edge of the target, is advancing than that of geometrical stagnation point, as shown in Fig. 9. The conclusions drawn by [40] agree with the observation that maximum Nusselt number is found at downstream of meeting of jet axis and target surface.

When micro analysis is performed, it is observed that for the water jet, the stagnation point is shifting to an upstream direction from the geometric centre of the jet $\left(X_{0}\right)$. For example, in a $2 \mathrm{~mm}$ wide slot jet, the stagnation point shifts by about $1.5 \mathrm{~mm}$ to the upstream side for the water jet at a 60 degree inclination. These observations are made by numerical analysis [33]. This may happen due to the use 


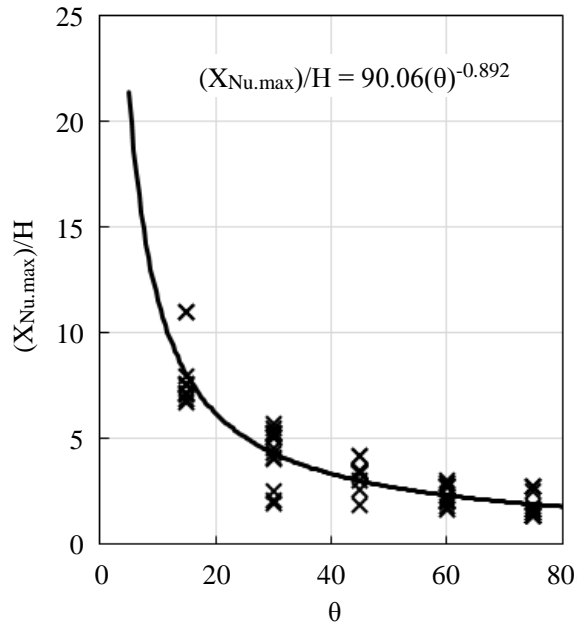

(a)

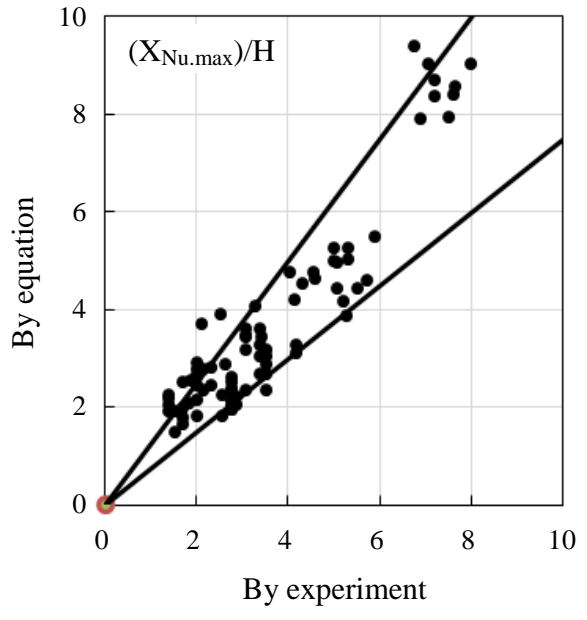

(b)

Fig. 8. Equation suggested for $X_{\text {Nu.max }}$ : a) variation w.r.t. jet inclination angle b) comparison with experimental and equation results

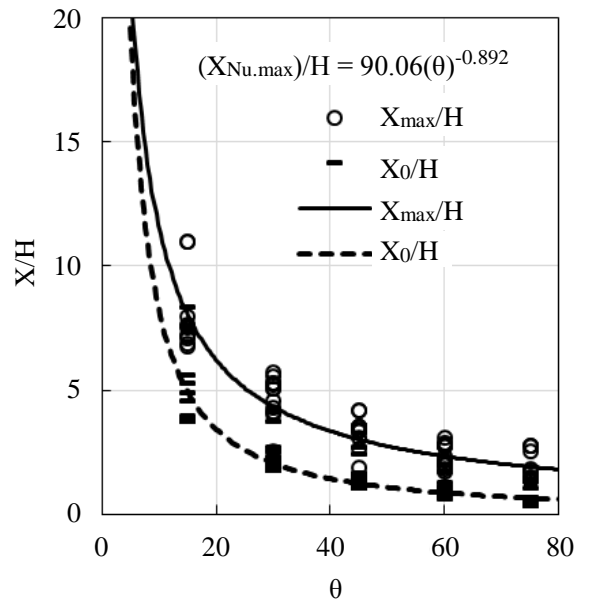

Fig. 9. Variation of $X_{\text {Nu.max }}$ and $X_{0}$ - related with jet inclination

of water as jet fluid, being of high density and viscosity, also as jet with dissimilar fluid (liquid + air) and higher adhesive forces between their molecules.

For an air jet specifically, this pattern is observed. For a liquid jet, the entrainment effect is smaller, but for an "air" jet the entertainment causes more jet spreading, as it is a submerged jet in alike fluid. Due to additional fluid consumption during entrainment, the average velocity of the jet landing decreases. This also causes an increase in the jet surface area which is called an impingement area, typically in the downside flow. The same reason may effect on the move in location of maximum Nusselt number to the downhill side of target plate. 


\section{Conclusion}

The position of the maximum Nusselt number analysis has been presented with reference to an area-based average. The local Nu distribution along the $\mathrm{Y}$ axis follows symmetry, and symmetry is seen to be unaffected by the changes in jet inclination. As the inclination angle decreases, the coolest points with maximum heat transfer shift to the downhill side of the target plate. The inclination of 45 and 60 degree show better cooling compared to other jet inclinations. Also, the Nusselt number increases with the Reynolds number, but the maximum Nusselt number position remains unchanged for a specific angle, and target-to-jet height. For the position of the maximum Nusselt number, the summary of the conclusion shows that at a higher angle ratio, the angle is the dominating parameter compared with the Reynolds number. At a lower angle ratio, the inclined jet with a higher Reynolds number gives the cooling point away from the leading edge. The cold spot position remains almost unchanged for a specific angle and target-to-jet height. Also, it is observed that for a particular angle of incident, the location of cold spot/maximum Nusselt number, measured from leading edge of target, is ahead of that of stagnation point in the stated condition.

Manuscript received by Editorial Board, August 30, 2017; final version, November 17, 2017.

\section{References}

[1] C.J.M. Lasance. Advances in high-performance cooling for electronics. Electronics Cooling, Nov. 1, 2006. https://www.electronics-cooling.com/2005/11/advances-in-high-performancecooling-for-electronics/.

[2] H. Metwally. Methods of evaluating advanced electronics colling systems. online: www.ansys.com, 2008.

[3] A. Bhattacharya and R.L. Mahajan. Metal foam and finned metal foam heat sinks for electronics cooling in buoyancy-induced convection. ASME. Journal of Electronic Packaging, 128(3):259266, 2006. doi: 10.1115/1.2229225.

[4] S. Sathe and B. Sammakia. A review of recent developments in some practical aspects of air-cooled electronic packages. ASME. Journal of Heat Transfer, 120(4):830-839, 1998. doi: $10.1115 / 1.2825902$.

[5] R.C. Chu. The challenges of electronic cooling: past, current and future. ASME. Journal of Electronic Packaging, 126(4):491-500, 2004. doi: 10.1115/1.1839594.

[6] J.-M. Koo, S. Im, L. Jiang, and K.E. Goodson. Integrated microchannel cooling for threedimensional electronic circuit architectures. ASME. Journal of Heat Transfer, 127(1):49-58, 2005. doi: $10.1115 / 1.1839582$.

[7] R. Dharmalingam, K.K. Sivagnanaprabhu, J. Yogaraja, S. Gunasekaran, and R. Mohan. Experimental investigation of heat transfer characteristics of nanofluid using parallel flow, counter flow and shell and tube heat exchanger. Archive of Mechanical Engineering, 62(4):509-522, 2015. doi: 10.1515/meceng-2015-0028.

[8] S.S. Anandan and V. Ramalingam. Thermal management of electronics: A review of literature. Thermal Science, 12(2):5-26, 2008. doi: 10.2298/TSCI0802005A. 
[9] M.-Y. Wen. Flow structures and heat transfer of swirling jet impinging on a flat surface with micro-vibrations. International Journal of Heat and Mass Transfer, 48(3):545-560, 2005. doi: 10.1016/j.ijheatmasstransfer.2004.09.010.

[10] P.A. Dellenback, J.L. Sanger, and D.E. Metzger. Heat transfer in coaxial jet mixing with swirled inner jet. ASME. Journal of Heat Transfer, 116(4):864-870, 1994. doi: 10.1115/1.2911460.

[11] S.K. Hong, D.H. Lee, and H.H. Cho. Heat/mass transfer measurement on concave surface in rotating jet impingement. Journal of Mechanical Science and Technology, 22(10):1952-1958, 2008. doi: 10.1007/s12206-008-0738-5.

[12] J.S. Bintoro, A. Akbarzadeh, and M. Mochizuki. A closed-loop electronics cooling by implementing single phase impinging jet and mini channels heat exchanger. Applied Thermal Engineering, 25(17):2740-2753, 2005. doi: 10.1016/j.applthermaleng.2005.01.018.

[13] B.P. Whelan, R. Kempers, and A.J. Robinson. A liquid-based system for CPU cooling implementing a jet array impingement waterblock and a tube array remote heat exchanger. Applied Thermal Engineering, 39:86-94, 2012. doi: 10.1016/j.applthermaleng.2012.01.013.

[14] C. Glynn, T. O’Donovan, and D.B. Murray. Jet impingement cooling. In Proceedings of the 9th UK National Heat Transfer Conference, Manchester, 2005.

[15] K.M. Graham and S. Ramadhyani. Experimental and theoretical studies of mist jet impingement cooling. ASME. Journal of Heat Transfer, 118(2):343-349, 1996. doi: 10.1115/1.2825850.

[16] J. Garg, M. Arik, S. Weaver, T. Wetzel, and S. Saddoughi. Meso scale pulsating jets for electronics cooling. ASME. Journal of Electronic Packaging, 127(4):503-511, 2005. doi: 10.1115/1.2065727.

[17] Y. Utturkar, M. Arik, C.E. Seeley, and M. Gursoy. An experimental and computational heat transfer study of pulsating jets. ASME. Journal of Heat Transfer, 130(6):062201, 2008. doi: 10.1115/1.2891158.

[18] S.C. Arjocu and J.A. Liburdy. Identification of dominant heat transfer modes associated with the impingement of an elliptical jet array. ASME. Journal of Heat Transfer, 122(2):240-247, 2000. doi: $10.1115 / 1.521463$.

[19] B.P.E. Dano, J.A. Liburdy, and K. Kanokjaruvijit. Flow characteristics and heat transfer performances of a semi-confined impinging array of jets: effect of nozzle geometry. International Journal of Heat and Mass Transfer, 48(3):691-701, 2005. doi: 10.1016/j.jheatmasstransfer.2004.07.046.

[20] B.P. Whelan and A.J. Robinson. Nozzle geometry effects in liquid jet array impingement. Applied Thermal Engineering, 29(11):2211-2221, 2009. doi: 10.1016/j.applthermaleng.2008.11.003.

[21] A. Pavlova and M. Amitay. Electronic cooling using synthetic jet impingement. ASME. Journal of Heat Transfer, 128(9):897-907, 2006. doi: 10.1115/1.2241889.

[22] H.S. Sheriff and D.A. Zumbrunnen. Effect of flow pulsations on the cooling effectiveness of an impinging jet. ASME. Journal of Heat Transfer, 116(4):886-895, 1994. doi: 10.1115/1.2911463.

[23] L.A. Brignoni and S.V. Garimella. Experimental optimization of confined air jet impingement on a pin fin heat sink. IEEE Transactions on Components and Packaging Technologies, 22(3):399-404, 1999. doi: 10.1109/6144.796542.

[24] D.H. Lee, Y.S. Chung, and P.M. Ligrani. Jet impingement cooling of chips equipped with multiple cylindrical pedestal fins. ASME. Journal of Electronic Packaging, 129(3):221-228, 2007. doi: $10.1115 / 1.2753884$.

[25] H. Eren and N. Celik. Cooling of a heated flat plate by an obliquely impinging slot jet. International Communications in Heat and Mass Transfer, 33(3):372-380, 2006. doi: 10.1016/j.icheatmasstransfer.2005.10.009. 
[26] M. Fabbri, S. Jiang, and V.K. Dhir. A comparative study of cooling of high power density electronics using sprays and microjets. ASME. Journal of Heat Transfer, 127(1):38-48, 2005. doi: 10.1115/1.1804205.

[27] K. Choo, T.Y. Kang, and S.J. Kim. The effect of inclination on impinging jets at small nozzleto-plate spacing. International Journal of Heat and Mass Transfer, 55(13):3327-3334, 2012. doi: 10.1016/j.ijheatmasstransfer.2012.02.062.

[28] K. Nakabe, E. Fornalik, J.F. Eschenbacher, Y. Yamamoto, T. Ohta, and K. Suzuki. Interactions of longitudinal vortices generated by twin inclined jets and enhancement of impingement heat transfer. International Journal of Heat and Fluid Flow, 22(3):287-292, 2001. doi: 10.1016/S0142-727X(01)00090-X.

[29] Y.-T. Yang and Y.-X. Wang. Three-dimensional numerical simulation of an inclined jet with cross-flow. International Journal of Jeat and Mass Transfer, 48(19):4019-4027, 2005. doi: 10.1016/j.ijheatmasstransfer.2005.04.018.

[30] B.Q. Li, T. Cader, J. Schwarzkopf, K. Okamoto, and B. Ramaprian. Spray angle effect during spray cooling of microelectronics: experimental measurements and comparison with inverse calculations. Applied Thermal Engineering, 26(16):1788-1795, 2006. doi: 10.1016/j.applthermaleng.2006.01.023.

[31] C. Bartoli. Free convection enhancement between inclined wall and air in presence of expired jets at temperature difference of 40 K. Experimental Thermal and Fluid Science, 35(2):283-290, 2011. doi: 10.1016/j.expthermflusci.2010.09.010.

[32] D. Benmouhoub and A. Mataoui. Inclined plane jet impinging a moving heated wall. Fluid Dynamics \& Materials Processing, 10(2):241-260, 2014. doi: 10.3970/fdmp.2014.010.241.

[33] A.Y. Tong. On the impingement heat transfer of an oblique free surface plane jet. International Journal of Heat and Mass Transfer, 46(11):2077-2085, 2003. doi: 10.1016/S00179310(02)00505-7.

[34] S. Ingole and K. Sundaram. Review of experimental investigation in heat transfer for jet impingement cooling. International Review of Mechanical Engineering, 6(3):346-356, 2012.

[35] S.H. Yoon, M.K. Kim, and D.H. Lee. Turbulent flow and heat transfer characteristics of a two-dimensional oblique plate impinging jet. KSME International Journal, 11(4):476-483, 1997. doi: 10.1007/BF02945086.

[36] S.B. Ingole and K.K. Sundaram. Experimental average Nusselt number characteristics with inclined non-confined jet impingement of air for cooling application. Experimental Thermal and Fluid Science, 77:124-131, 2016. doi: 10.1016/j.expthermflusci.2016.04.016.

[37] S.B. Ingole and K.K. Sundaram. Heat transfer enhancement factor characteristics for collective cooling using inclined air jet. In IEEE 17th Electronics Packaging and Technology Conference (EPTC), pages 1-6, Singapore, 2-4 Dec. 2015. IEEE. doi: 10.1109/EPTC.2015.7412395.

[38] A. Ianiro and G. Cardone. Heat transfer rate and uniformity in multichannel swirling impinging jets. Applied Thermal Engineering, 49:89-98, 2012. doi: 10.1016/j.applthermaleng.2011.10.018.

[39] C.F. Ma, Q. Zheng, H. Sun, K. Wu, T. Gomi, and B.W. Webb. Local characteristics of impingement heat transfer with oblique round free-surface jets of large Prandtl number liquid. International Journal of Heat and Mass Transfer, 40(10):2249-2259, 1997. doi: 10.1016/S00179310(96)00310-9.

[40] N. Zuckerman and N. Lior. Jet impingement heat transfer: physics, correlations, and numerical modeling. Advances in Heat Transfer, 39:565-631, 2006. doi: 10.1016/S0065-2717(06)390065.

[41] A. Ramezanpour, H. Shirvani, and I. Mirzaee. A numerical study on the heat transfer characteristics of two-dimensional inclined impinging jet. In 5th Electronics Packaging Technology Conference (EPTC), pages 626-632, Singapore, 12 Dec. 2003. IEEE. doi: 10.1109/EPTC.2003.1271594. 